\title{
Association between anthropometric characteristics and bladder cancer risk: a systematic review and meta-analysis of longitudinal cohort studies
}

Lazaros Tzelves ${ }^{1}$, Dimitra Xenou ${ }^{2}$, Andreas Skolarikos ${ }^{1}$, Ioannis Varkarakis ${ }^{1}$, Charalampos Deliveliotis $^{1}$, Evangelos Terpos ${ }^{2}$, Kimon Stamatelopoulos ${ }^{2}$, Theodoros Sergentanis ${ }^{2}$, and Theodora Psaltopoulou ${ }^{2}$

${ }^{1}$ Geniko Nosokomeio Attikes Sismanogleio Amalia Phlemink

${ }^{2}$ National and Kapodistrian University of Athens

July 13, 2020

\begin{abstract}
Background/ Aims Anthropometric characteristics, namely overweight/obesity, height and waist circumference have been associated with various cancer types. The aim of this systematic review and meta-analysis is to evaluate the potential associations between anthropometric characteristics and bladder cancer risk, synthesizing longitudinal cohort studies. Methods Literature search across MEDLINE, EMBASE, Scopus, Google Scholar and Cochrane Central was performed up to December 31,2019 and data abstraction was performed independently by two authors. Random-effects (DerSimonian-Laird) models were used to estimate pooled relative risks (RR) and 95\% confidence intervals (95\% CI); subgroup analyses were performed geographical region, mean age, publication year, length of follow-up, sample size, method of BMI estimation and adjustment for smoking. Results A total of 27 studies were included (88,593 bladder cancer cases in a total cohort of 49,647,098 subjects). Increased bladder cancer risk was noted in overweight men (pooled $\mathrm{RR}=1.12,95 \% \mathrm{CI}$ : 1.04-1.21) but not in overweight women. Both obese men (pooled $\mathrm{RR}=1.14,95 \% \mathrm{CI}: 1.06-1.22$ ) and women (pooled $\mathrm{RR}=1.19,95 \% \mathrm{CI}$ : 1.02-1.38) showed increased risk. Interestingly, height increase per $5 \mathrm{~cm}$ did not seem to affect risk of bladder cancer in men (pooled RR=1.03, 95\%CI: 0.99- 1.06) and women (pooled $\mathrm{RR}=1.02,95 \% \mathrm{CI}$ : 0.97-1.06). Larger waist circumference was associated with bladder cancer risk in men (pooled $\mathrm{RR}=1.18,95 \% \mathrm{CI}$ : 1.09-1.26) but not women. Conclusion In conclusion, bladder cancer risk seems to be related with obesity overall and central obesity in men. In contrast to other cancer types, height does not seem to affect risk, but more studies are needed to extract safe conclusions.
\end{abstract}

\section{Association between anthropometric characteristics and bladder cancer risk: a systematic review and meta-analysis of longitudinal cohort studies.}

Lazaros Tzelves $^{1}$, Dimitra Xenou ${ }^{2}$, Andreas Skolarikos ${ }^{1}$, Ioannis Varkarakis ${ }^{1}$, Charalampos Deliveliotis ${ }^{1}$, Evangelos Terpos ${ }^{2}$, Kimon Stamatelopoulos ${ }^{2}$, Theodoros N. Sergentanis ${ }^{2} *$, Theodora Psaltopoulou ${ }^{2} *$

* The two authors contributed equally

1: 2nd Department of Urology, Sismanoglio Hospital, Medical School, National and Kapodistrian University of Athens, Sismanogliou 37 Athens, Greece.

2: Department of Clinical Therapeutics, "Alexandra" Hospital, Medical School, National and Kapodistrian University of Athens, 80 Vas Sofias Ave, Athens 11528, Greece.

Corresponding author : Lazaros Tzelves, lazarostzelves@gmail.com, 2nd Department of Urology, Sismanoglio Hospital, Medical School, National and Kapodistrian University of Athens, Sismanogliou 37 Athens, Greece 
Conflicts of Interest: The authors declare that they have no potential conflict of interest Funding: No funding was received

\section{Hosted file}

manuscript.docx available at https://authorea.com/users/342152/articles/469025-associationbetween-anthropometric-characteristics-and-bladder-cancer-risk-a-systematic-review-andmeta-analysis-of-longitudinal-cohort-studies

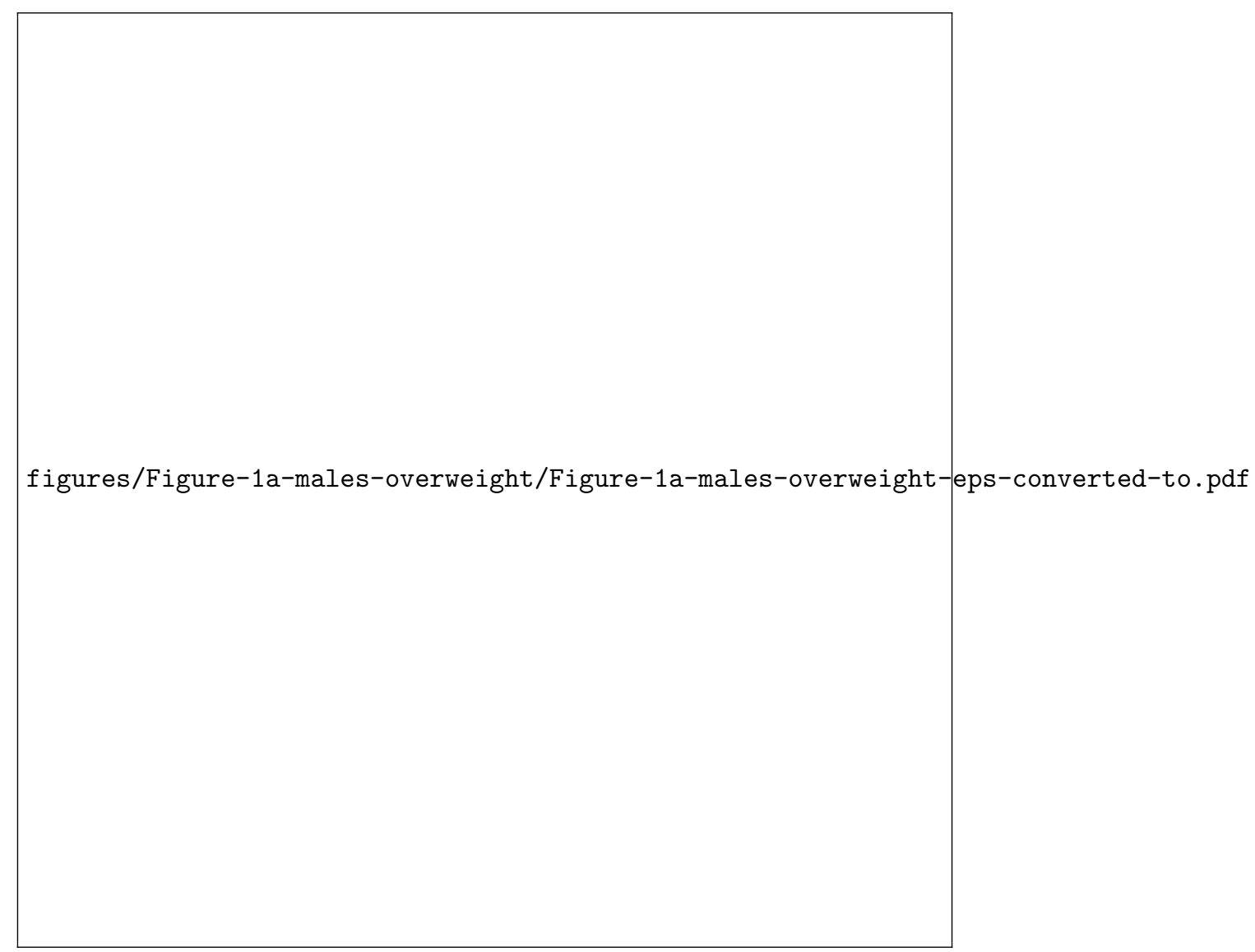


figures/Figure-1b-males-obese/Figure-1b-males-obese-eps-converted-to.pdf 
figures/Figure-1c-females-overweight/Figure-1c-females-overweight-eps-converted-to.pdf 
figures/Figure-1d-females-obese/Figure-1d-females-obese-eps-converted-to.pdf 


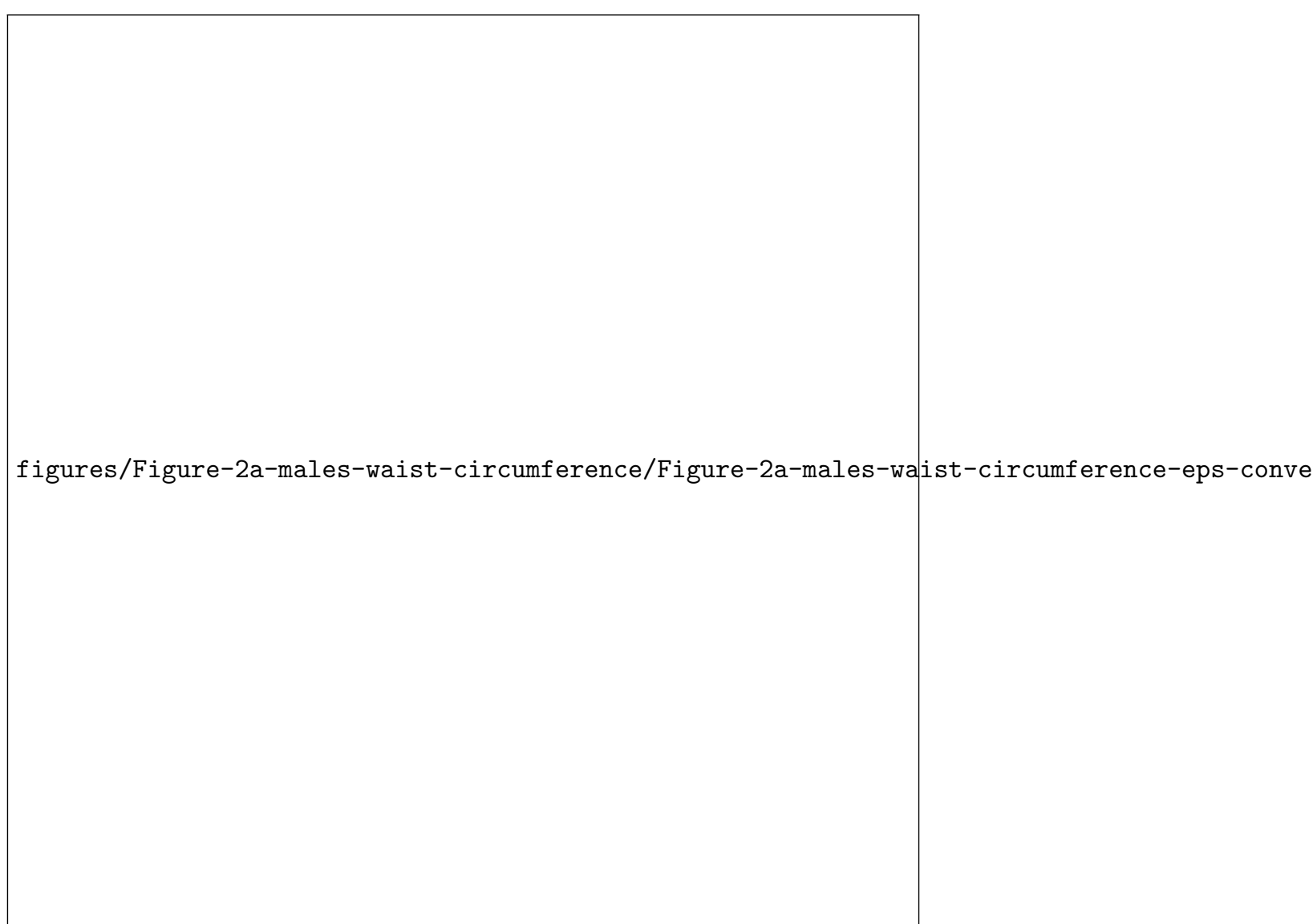




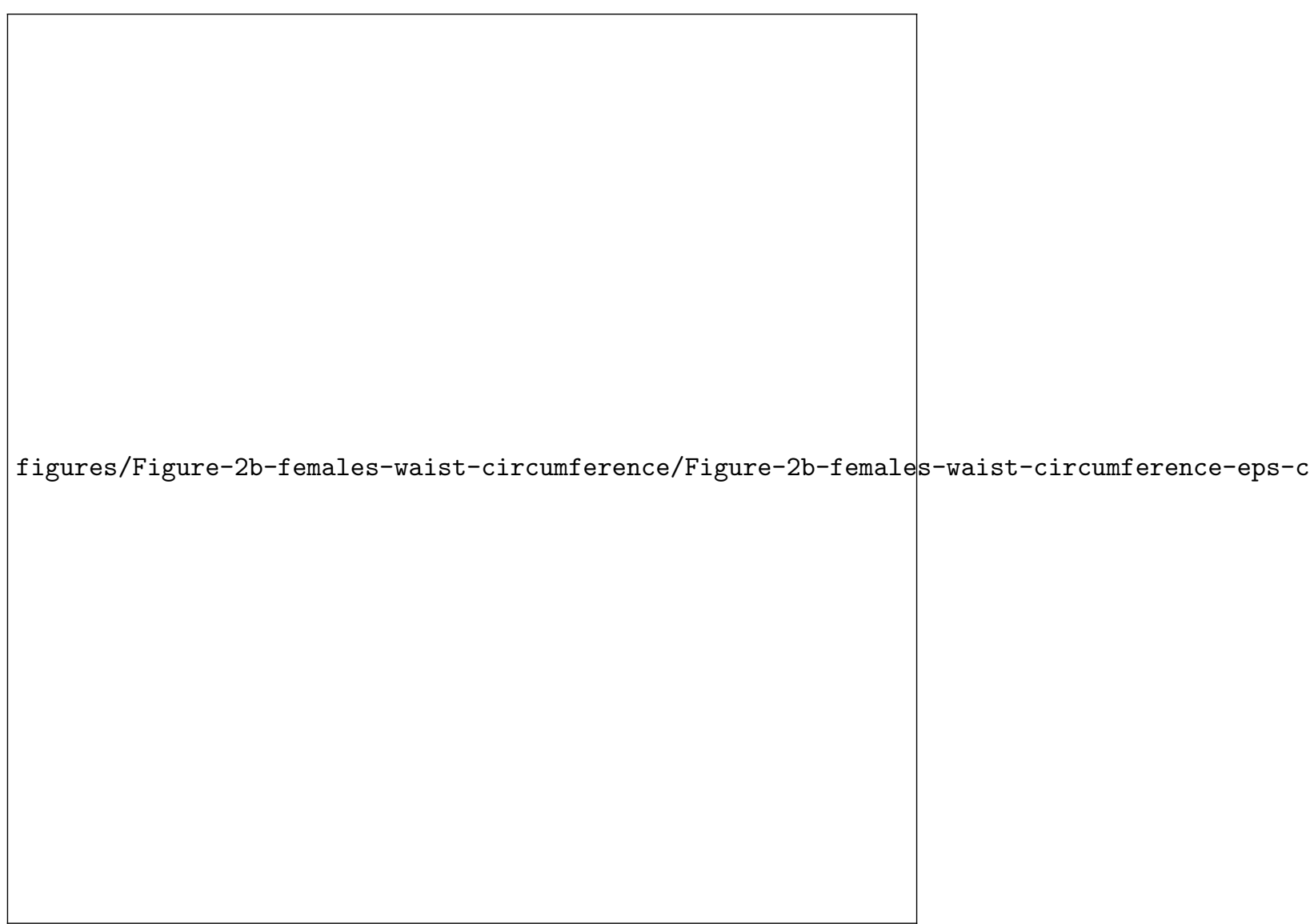

